\title{
HACIA UNA TEORÍA DE LOS BIENES PÚBLICOS COMO BIENES POLÍTICOS Y EL ROL ESTATAL
}

\author{
ALAN G. FUTERMAN*
}

Fecha de recepción: 13 de noviembre de 2013.

Fecha de aceptación: 20 de marzo de 2014.

Resumen: En el presente trabajo se realiza un análisis de los Bienes Públicos desde su definición de «No Exclusión» y «No Rivalidad» para demostrar las contradicciones en que incurre la misma. A su vez se propone como término correcto el de Bienes Políticos para ilustrar que aquellas funciones que el Estado adopta por encima de las básicas (seguridad y justicia) son fruto de presiones políticas y la arbitrariedad de los funcionarios (lo cual es ilustrado con las teorías de James Buchanan sobre la Democracia). Finalmente se procede a brindar una posible justificación de la provisión estatal de Seguridad y Justicia bajo los fundamentos de la Teoría Económica (en base a las teorías de Robert Nozick), lo cual demostraría que estos son bienes para cuya provisión el Estado podría ser el agente o árbitro ideal, sin necesidad de recurrir a la categoría contradictoria y ambigua de Bienes Públicos.

Palabras clave: Bienes Públicos, Praxeología, Ludwig von Mises, Escuela de la Elección Pública, James Buchanan, Estado Mínimo, Robert Nozick, Orden Espontáneo, Orden Creado, Externalidades.

Clasificación JEL: H40, H41, H42, D62.

Abstract: In this work Public Goods are analyzed from the point of view of their definition as «non-excludable» and «non-rivalry» in order to show the contradictions it entails. Also we propose Political Goods as a proper name in order to define those goods provided by the state apart from the basic ones (security and justice) and therefore to show how these are the result of political pressure and arbitrary decisions by bureaucrats (which is studied in the works of James Buchanan on Democracy). Finally we present a possible justification

\footnotetext{
* Universidad del Centro Educativo Latinoamericano, Investigador - Fundación Bases, Editor - www.catalactica.com.ar. Santa Fe, Argentina.
} 
for the provision of Security and Justice by the State based on Economic Theory (according to the theories of Robert Nozick), which would show that the State could be the ideal agent to provide these goods, while at the same time avoid using the contradictory and ambiguous category of Public Goods.

Key words: Public Goods, Praxeology, Ludwig von Mises, Public Choice School, James Buchanan, Minimal State, Robert Nozick, Spontaneous Order, Created Order, Externalities.

JEL Classification: H40, H41, H42, D62.

\section{I INTRODUCCIÓN ${ }^{1}$}

Múltiples autores han tratado el estudio de los llamados «Bienes Públicos» y han analizado los mismos desde diversos puntos de vista, tanto económicos como desde la filosofía política. ${ }^{2}$ Sin embargo no es extraño observar que aún hoy, cuando ya mucho se ha escrito al respecto, continúa un manto de duda sobre el propio concepto de Bienes Públicos y su marco de aplicación a la hora de designar como tales a ciertos bienes en la realidad.

Las características resaltadas como representativas de los Bienes Públicos son la «No Exclusión» y la «No Rivalidad» en el consumo. Esto es, que no se puede excluir a ningún individuo del consumo del bien en cuestión, y a la vez que el consumo de $n$ unidades por parte de un individuo no implica que otro no podrá consumirlo, respectivamente. Un simple análisis de los bienes que podrían definirse como tales torna evidente que la definición de Bienes Públicos con estas características hace de la misma lo suficientemente ambigua como para no demarcar el concepto con la rigurosidad necesaria.

La rigurosidad y la necesidad de una definición exenta de vaguedad y ambigüedad es necesaria puesto que los Bienes Públicos surgirían como una necesidad objetiva básica en una sociedad

${ }^{1}$ Una versión de este trabajo ha sido presentado como ensayo final de la materia Economía de las Instituciones en la Universidad del Centro Educativo Latinoamericano, dictada por el profesor Walter Castro a quién le agradezco sus valiosos comentarios y aportes.

2 Entre otros ver de Jasay, A. ([1985] 1998). 
moderna fruto de la supuesta «Falla de Mercado» en la provisión de los mismos. Por ejemplo la construcción, mantenimiento y control de calles.

Por lo tanto, los Bienes Públicos debieran de ser definidos con exactitud de forma tal de permitir identificar cuáles son aquellos bienes que el Estado debe proveer, ya que según se afirma, el mercado no los provee. A la hora de efectuar el análisis de cuáles son estos bienes, es donde se dificulta su definición. Esto ocurre puesto que la definición de que son los Bienes Públicos puede responder a diversos criterios:

a) Aquellos bienes que el Mercado no provee.

b) Aquellos bienes que el Mercado provee, pero ineficientemente.

c) Aquellos bienes que el Mercado no puede proveer.

d) Aquellos bienes que el Estado puede proveer.

e) Aquellos bienes que el Estado debería proveer.

f) Aquellos bienes que solo el Estado debe proveer.

Como vemos, en los primeros tres casos la definición de Bienes Públicos parte de hechos de carácter negativo, lo que el Mercado no provee, provee ineficientemente o no puede proveer. Ante esto es interesante notar ciertos puntos. Constituye un non sequitur afirmar que el Estado debe proveer un bien que el Mercado no provee solo porque este no lo provea. Que el Mercado provea algo ineficientemente implica definir primero que se entiende por eficiencia y posteriormente determinar por qué razón se supone que el Estado lo proveerá más eficientemente. Y por ultimo si se afirma que el Mercado no puede proveer un determinado bien (o bienes), debe explicarse porque es este el caso y porque sí solo el Estado está facultado para proveerlo.

En los últimos tres casos la definición no sería en relación al Mercado, sino que afirmaría positivamente que son los Bienes Públicos, esto es, son aquellos que el Estado puede o debería proveer, o aquellos que solo el Estado estaría facultado a proveer. Sin embargo nuevamente deberíamos, antes que cualquier análisis ulterior, conocer cuál es la razón de ser, naturaleza y funciones específicas del Estado, puesto que no podemos saber que debe proveer el Estado si no es que antes suponemos que debería existir. Por 
otro lado no podríamos saber que puede proveer si no conocemos su naturaleza y funciones específicas. Finalmente deberíamos determinar por qué sería el Estado el único facultado para la provisión de determinados bienes y no el mercado.

Aún así, y si bien este marco de análisis de la definición de Bienes Públicos es breve, vemos sin más que la definición de «No Rivalidad»y «No Exclusión» difícilmente haga luz a todos estos interrogantes. Precisamente la ambigüedad y vaguedad de la definición permite que pueda incluirse prácticamente cualquier bien en la categoría de Bienes Públicos. Debido a que no hay una definición especifica, el propio concepto de Bienes Públicos lleva indefectiblemente a la expansión de las atribuciones estatales, puesto que cualquier bien que se identifique que «El Mercado no puede proveer», «El Mercado no provee eficientemente», «El Mercado no provee», «El Estado debería proveer», «El Estado puede proveer» y/o «Solo el Estado debe proveer» (estas razones pueden usarse singular o conjuntamente) lleva a que el Estado monopolice su provisión (ergo, que se expanda).

Así vemos que la vaguedad conceptual en la definición de los Bienes Públicos es una de las bases del crecimiento del Estado, y a su vez de su legitimación (en el discurso político). A su vez así se justifica su expansión, sobre la base de la provisión de nuevos y crecientes Bienes Públicos.

Al respecto del rango de aplicación de la definición de Bienes Públicos, Paul Samuelson ha llegado a afirmar:

Thus, consider what I have given in this paper as the definition of a public good, and what I might better have insisted upon as a definition in my first and subsequent papers: «A public good is one that enters two or more persons' utility.» What are we left? Two poles and a continum in between? No. With a knife-edge pole of the private-good case and with all the rest of the world in the public-good domain by virtue of involving some «consumption externality». ${ }^{3}$

A continuación no analizaremos por que el Mercado podría (e incluso sería preferible de acuerdo a algunos autores $)^{4}$ proveer

${ }^{3}$ En Samuelson ([1969] 1991, 502).

4 Ver Murphy ([2002] 2010), entre otros. 
aquellos bienes que hoy provee el Estado, con mayor justicia y eficiencia; ni tampoco analizaremos el origen y evolución de las atribuciones estatales a este respecto, sino que nos concentraremos en mostrar como el propio concepto de Bienes Públicos (en base a la definición de «No Exclusión» y «No Rivalidad») implica una contradicción que impide e impedirá que se sepa exactamente que bienes han de ser incluidos en el ámbito de los Bienes Públicos, y por tanto mediante la ambigüedad conceptual se perpetúe la expansión estatal.

\section{II}

\section{ANÁLISIS DE LA DEFINICIÓN DE BIENES PÚBLICOS A LA LUZ DE LA PRAXEOLOGÍA}

A la hora de analizar la definición de Bienes Públicos en relación a sus características distintivas es relevante utilizar el herramental teórico formal que provee la Praxeología ${ }^{5}$ para identificar su relevancia y pertinencia en la teoría económica. Para ello nos basaremos en el razonamiento, meramente formal, de la Ley de Rendimientos Decrecientes que realiza Ludwig Von Mises (por el llamada «Ley de Rendimientos»), en su opus magnum «La Acción Humana», 6 para poder ser capaces de corroborar si tal definición se corresponde con los postulados de la teoría económica o no, $y$, por tanto saber si la misma es correcta para describir la realidad y la prescripción de Políticas Públicas.

Mises afirma que dicha Ley no es fruto de la experiencia (sin importar cuán cierta resulte en el ámbito técnico de específicos procesos de producción), sino una deducción a priori, implícita en la categoría de Acción Humana. Comienza su razonamiento afirmando que en el caso de Bienes de Primer Orden, ${ }^{7}$ una cantidad $a$

${ }^{5}$ La Ciencia de la Acción Humana, basada en el Axioma de la Acción y sus implicancias lógicas. Para una descripción detallada ver Mises ([1949] 1986).

6 Mises ([1949] 1986, 205-210).

7 Carl Menger ([1871] 2007) distinguió a los bienes económicos en órdenes inferiores y superiores, en relación a su cercanía al consumo. Esto es, los bienes de orden inferior (o primer orden) son los bienes de consumo y aquellos de orden superior (o segundo, tercer, cuarto,...n órdenes) son aquellos que se utilizan en los procesos de 
de causa produce una cantidad $\alpha$ de efecto (mediata o inmediatamente). En el caso de Bienes de Orden Superior una determinada cantidad $b$ del bien B produce una determinada cantidad de efecto $\beta$, mientras que una determinada cantidad $c$ del bien $C$ produce una determinada cantidad de efecto $\Upsilon$. Los efectos conjuntos de $\beta$ y $\Upsilon$ de ambos bienes complementarios producen la cantidad $p$ del bien de primer orden D. Si $b$ es fijo, el valor de $c$ óptimo será aquel donde la relación $p / c$ sea más grande. Si más de un valor de $c$ implica el más alto valor de $p / c$ entonces el óptimo será aquel donde $p$ sea mayor. Así, el mayor rendimiento se da cando ambos factores se utilizan en su cuantía óptima. Un incremento en la cuantía de uno de los factores genera un mayor rendimiento pero en una proporción menor que el incremento de dicho factor, de lo contrario no podríamos hablar de un óptimo en la relación anterior.

Por ejemplo, si pudiesen reemplazarse mayores unidades de $c$ por menos unidades de $b$ al mismo tiempo que se mantiene constante la misma producción de $p$ entonces $B$ sería un bien de producción ilimitada (siempre y cuando haya suficiente cuantía disponible de $C$ ), y por tanto no sería un bien económico puesto que no resulta escaso. En tal caso sería un bien libre.

En sentido inverso, si aumentase la cuantía de $b$ pero la cuantía producida de $p$ permanecería constante entonces el aumento de $p$ sería enteramente debido al aumento de $c$, por lo que $B$ no sería un bien económico.

La Ley de Rendimientos Decrecientes por tanto afirma que en el caso de bienes de Orden Superior hay combinaciones óptimas de factores que producen una cuantía mayor de producción, y cuando hay un desvío en el consumo de uno de los factores entonces disminuirá el crecimiento de la cuantía producida o crecerá menos que proporcionalmente al mayor consumo del factor de producción (de Orden Superior) utilizado. La clave del razonamiento es que los efectos cuantitativos del consumo del factor en relación a la cuantía producida de $p$ deben ser limitados, y por ello recibir el nombre de bienes económicos.

producción de bienes de primer orden, clasificados de acuerdo a cuan alejados están de la etapa final de consumo. 
En el caso de los Bienes Públicos se habla de «No Rivalidad» $\mathrm{y}$ «No Exclusión». Sin embargo, como veremos esto implica graves contradicciones.

Veamos el razonamiento lógico (praxeológico): Los bienes libres no son escasos y por lo tanto su utilidad marginal es 0 , lo cual implica que los individuos no requieren recurrir a esfuerzos o comportamientos deliberados a los efectos de obtener tales bienes o proceder a su consumo (su valor es nulo porque existe suficiente cantidad para cubrir la demanda existente). Los bienes económicos si son escasos y su utilidad marginal es mayor a 0 , esto es, su cuantía resulta en una disponibilidad insuficiente para satisfacer la demanda de los fines individuales. Por lo tanto los individuos incurren en esfuerzos y comportamientos deliberados para obtener tales bienes y utilizarlos como medios para la satisfacción de determinados fines.

Una vez tenida en cuenta la distinción anterior, el análisis de las condiciones de «No Exclusión» y «No Rivalidad» a la luz del herramental formal de la Praxeología resultará fundamental a la hora de identificar la validez o invalidez del concepto de Bienes Públicos. Veamos:

Si existe una disponibilidad de una cantidad $c$ del bien $A$ y dos individuos, $Z$ e $Y$, lo requieren para la prosecución de sus fines, entonces si $Z$ tiene acceso a una unidad del bien $A$ la cuantía del mismo disminuye a $c-1$. Consecuentemente $Z$ utiliza dicha unidad para la satisfacción del fin más inmediato, y por tanto se produce un incremento en la utilidad marginal de las unidades disponibles de $A$ (ahora de cuantía $c-1$ ) para el individuo $Y$, que ahora no podrá satisfacer el último fin al que deseaba satisfacer con dicha unidad que no pudo obtener.

Por tanto como la cantidad de $c$ no es $\infty$ (infinita), puesto que la cuantía disponible es ahora $c$ - 1 , entonces (si no hay un aumento en la producción o existencia disponible de $c$ ) el consumo de $a$ unidades de $A$ por parte de $Z$ implica que habrá $a$ unidades de $A$ menos para el consumo de $Y$. Si, por el contrario, ante un consumo de $a$ unidades de $A$ por parte de $Z$, la utilidad marginal de $A$ para $Y$ no disminuiría, implicaría que habría suficientes $o \infty$ (infinitas) unidades de $A$ disponibles para la satisfacción de todos los fines de $Y$. Sin embargo en este caso, a diferencia del primero, estaríamos 
hablando de un bien libre y no de un bien económico. Por lo tanto la condición de «Rivalidad» en el consumo es una condición necesaria de la categoría de bienes económicos (lo cual quedó ilustrado en el primer caso mencionado).

En el mismo sentido, supongamos la existencia de una cantidad $d$ del bien $D$ y los individuos $G$ y $H$. Ambos proceden a adquirir unidades de $D$ sin que disminuyera $d$ (o que la disminución de $d$ sea lo suficientemente insignificante como para no lograr identificarse). En tal caso también hablaríamos, por tanto, de un bien libre y no de un bien económico. En este sentido la condición de «No Rivalidad» en el consumo es condición posible y necesaria solo en bienes libres.

Supongamos ahora la existencia de una cuantía del bien $U$ y los individuos $L$ y $K$. Si $L$ y $K$ consumieran la misma unidad de $U$ sin disminuir su cuantía total, implicaría que la cuantía de $U$ es $\infty$ (infinita), y por tanto hablaríamos de un bien libre. Si, por el contrario, el consumo simultáneo por parte de ambos individuos de una unidad de $U$ llevara a su cuantía total $q$ a $q$ - 1, hablaríamos de un bien económico, donde eventualmente los sucesivos consumos simultáneos de las restantes unidades de $U$ por parte de los individuos $L, P, \tilde{N}, S, \ldots n$ (siendo $n$ el total de individuos que consumen la cuantía total de q) llevarían eventualmente a la cuantía de $q$ a $q=0$ y por tanto imposibilitarían que otros individuos consumiesen más unidades de la ahora inexistente cuantía $q$ del bien $U$. En tal caso se trataría de un bien económico. Así, la condición de «No Rivalidad» es una condición posible y necesaria en bienes libres, no bienes económicos. Esto es así puesto que podría concebirse una situación donde el consumo de un bien es rival con respecto a uno o más individuos, aún cuando fuera que para que ello ocurra debería darse el hecho de que se agota la cantidad $q$ del bien $U$. En este sentido, la provisión de bienes «No Rivales» es irrelevante puesto que serían bienes libres.

En cuanto a la «No Exclusión», baste decir que la definición de derechos de propiedad (y su respeto efectivo) es el mecanismo a partir del cual es posible excluir. ${ }^{8} \mathrm{Si}$ bien deben desarrollarse en

8 Ver Foldvary (2003). 
ciertos casos determinados mecanismos más complejos que otros para excluir, esto no implica que existan bienes que el propietario no pueda excluir de su consumo a otros (o que no podrá hacerlo en el futuro). Por otro lado, si por «No Exclusión» nos referiremos a bienes que por cuestiones políticas no puede excluirse de su consumo a individuos que no pueden acceder a ellos por sus propios medios, entonces nos alejamos del marco de la economía. Pero aquí no debiéramos utilizar conceptos propios de dicha disciplina, tales como el principio de Exclusión, ya que estaríamos extrapolando conceptos a otro campo. En este contexto, «No Exclusión» se confunde con Derechos (y en este caso, los llamados Derechos de Segunda y Tercera Generación, tales como sociales y ecológicos respectivamente).

Ahora bien, si los Bienes Públicos son aquellos cuyas características distintivas son las de «No Exclusión» y «No Rivalidad», entonces necesariamente implica contradicciones que tornan imposible identificar a que bienes se refiere en la realidad. Esto es así puesto que el análisis previo, basado en las herramientas de la Praxeología, nos permite distinguir que tales características responden únicamente o a bienes libres o a bienes seleccionados arbitrariamente por la política.

Sin embargo, los bienes libres no necesitan ser provistos por nadie, precisamente porque son no rivales y no excluibles en el consumo. Sería irrelevante que el Estado (así como el Mercado) se encargasen de la provisión de tales bienes precisamente porque ambas instituciones operan como mecanismos de provisión de aquellos bienes que son económicos (ergo rivales en el consumo y con posibilidad de exclusión) y deben, por tanto, ser producidos.

III

\section{POSIBLES CRÍTICAS A ESTE ENFOQUE}

La pregunta que podría elevarse luego de haber analizado lo expuesto anteriormente es: ¿Es posible que existan bienes económicos que al mismo tiempo se tornen no rivales o no excluibles en el consumo, y por tanto sean en parte (o se constituyan completamente) bienes libres (o viceversa)? Y en caso de que la respuesta sea afirmativa (lo 
cual devolvería legitimidad a la definición de Bienes Públicos), ¿Cómo deberían ser tratados? Para responder a este enfoque debemos citar nuevamente a Mises:

Un factor capaz de proporcionar tales ilimitados servicios es, por ejemplo, el conocimiento de cualquier relación de causalidad. La fórmula, la receta, que nos enseña a condimentar el café, una vez conocida, rinde servicios ilimitados. Por mucho que se emplee, nada pierde de su capacidad de producir; estamos ante una inagotable capacidad productiva, la cual, consecuentemente, deja de ser bien económico. Por eso nunca se halla el individuo actuante ante el dilema de tener que optar entre el valor en uso de una fórmula comúnmente conocida y el de cualquiera otra cosa útil. ${ }^{9}$

Aquí observamos que hay determinados bienes que una vez producidos (y disponibles indefinidamente para el individuo actuante) se convierten en bienes libres. Esto implica que antes de ser producidos implicaron el uso de bienes económicos (de los órdenes que sean) en la búsqueda de su creación, pero una vez creados su utilización no agrega ni saca nada en futuros procesos productivos mas allá de su aporte al momento inicial (siendo este bien de orden superior). Podemos decir que tales bienes son necesarios para la producción de otros bienes económicos, pero su aporte a la cuantía producida (sea cual sea) se completa y agota en el mismo momento del inicio del proceso de producción. El caso de la fórmula o la receta son ejemplos ilustrativos del caso. Así estos se convertirían en bienes libres. $O$, más precisamente, su efecto los llevaría a ser considerados como tales, y por tanto estos serán producidos (y consecuentemente el Mercado, si son demandados, los proveería), si el beneficio total percibido por los productores sea superior al costo de su producción (incluyendo el consumo sin contraprestación por parte de free riders).

Aquí debemos aclarar que disentimos con Mises puesto que incluso la receta o la fórmula operan como bienes económicos según la demostración expuesta en el apartado anterior puesto que eventualmente, por ejemplo, la transmisión de la información

9 Ver Mises ([1949] 1986, 206-207). 
de la receta a otros individuos que la desconocen implica incurrir en acciones con esfuerzos y propósitos deliberados junto a la selección de medios, esto es, la búsqueda de la receta y la reproducción de la misma en otra superficie. ${ }^{10}$ Que la receta sea relativamente menos escasa a otros bienes materiales no implica que sea un bien libre (que son del todo no escasos). Ya que la receta no es más que un concepto o un bien inmaterial (para ilustrar mejor lo que se pretende decir), se requiere de una superficie donde reproducirla para que esta pueda ser utilizada por otros individuos que no tienen acceso directo a la superficie donde actualmente se encuentra escrita (o grabada, etc.), y es precisamente dicha superficie la cual es limitada y sujeta a las antedichas condiciones de los bienes económicos. Los bienes inmateriales no se trasladan en el éter por su propia fuerza, sino que deben ser transmitidos a través de algún medio, y dicho medio será o implicará el uso de bienes económicos (por ejemplo por más que la receta se transmita por medio de la palabra se debe utilizar por ejemplo un teléfono para comunicarla, o si es presencialmente el individuo que la comunica debe haberse trasladado por algún medio hacia allí, incurriendo en un costo, aun sea el de caminar y no haber hecho otra cosa).

Veamos un ejemplo más difundido: El caso del Faro. El Faro ha sido definido como un Bien Público precisamente porque es supuestamente no excluible y no rival en su consumo. El hecho de que desde un Barco pueda verse la luz emitida por el Faro no impide que desde otro Barco lo hagan (tanto si ha pagado como si no el servicio del Faro), y los dueños del Faro no pueden impedir que desde ningún barco, pague o no, se vea la luz. Aquí es necesario hacer algunas aclaraciones. En primer lugar no es el Faro el que está siendo discutido como bien económico o libre (de hecho es un bien económico) sino el efecto del Faro, es decir, la luz.

${ }^{10}$ Es decir que un factor productivo con dichas características puede llegar a tener una productividad marginal nula en un determinado proceso productivo sin implicar esto que su productividad marginal no sea positiva en otros procesos de producción, por lo que lo primero no indica que deje de ser un bien económico. De lo contrario se podría afirmar que el capital fijo sería un bien no económico, lo cual sería absurdo. 
Como en el ejemplo de Mises arriba citado, una vez producido el bien (el Faro) y utilizado (encendida la luz), su efecto opera como un bien libre. Aún cuando podríamos identificar casos donde una gran cantidad de barcos tornasen que el consumo del Faro pueda ser rival en su consumo, esto no suele ocurrir puesto que su escasez es pequeña en relación a la demanda y el rango de alcance de su efecto (la luz). Sin embargo lo que es relevante a este respecto es que el Faro es un bien económico con un efecto que puede operar como un bien libre (tal como en el caso de la receta o fórmula) una vez que fue construido ( $y$ de ahi es que puede definirse como «No Rival»). Por lo tanto no invalida el razonamiento anterior sobre los bienes libres y económicos. Aquellos bienes que son económicos (y demandados) tendrán un incentivo de ser producidos, y por tanto tenderán a ser producidos por el Mercado. Pero incluso en este caso, como demuestra Ronald Coase, es posible su provisión por parte de privados y es posible la exclusión (aún cuando se defina como Bien Público). ${ }^{11}$

Teniendo en cuenta la salvedad anterior, y suponiendo que existen bienes libres que puedan tornarse en económicos, ${ }^{12}$ esto ocurriría si operase un factor externo que convirtiese en rival y/o excluible el consumo de dicho bien por parte de uno o más individuos, entonces estaríamos nuevamente en presencia de bienes económicos. Por ejemplo el caso de patentes, ya que una vez creada una fórmula (por citar un posible caso) su reproducción no implica que mengue su productividad (por lo que se constituiría en un bien libre, amén de que para su producción se requirieron bienes económicos como se ha dicho anteriormente), pero al implantarse el límite legal a su reproducción consecuentemente se lo torna en un bien escaso y por tanto en excluible (y si hay limites o cupos de uso, también en rival con respecto a su consumo). Lo que podemos deducir de lo anterior es que un bien libre (o cuyo

${ }^{11}$ El caso de los free riders fue analizado por Ronald Coase. Ver Foldvary (2003).

12 No se pone en duda la posibilidad de que un bien que sea considerado libre (como el aire) en un contexto determinado pueda ser un bien económico en otra circunstancia (por ejemplo en un escenario hipotético donde una contaminación catastrófica del aire generase la necesidad de que los individuos respirasen a través de tubos de oxigeno), sino el hecho de que algunos bienes sean ambiguos en cuanto a su definición de libres o económicos en un mismo contexto. 
efecto opera como de bien libre) solo puede ser considerado económico si existe una restricción externa que lo torne rival y/o excluible en cuanto a su consumo por parte de uno o más individuos.

Ahora bien, si la categoría de Bienes Públicos es tan ambigua y sus condiciones de «No Rivalidad» y «No Exclusión» incurren en contradicciones, surge la pregunta: ¿de donde proviene el criterio para seleccionar cuales son los Bienes Públicos en la realidad?

\section{LOS BIENES PÚBLICOS Y LOS BIENES POLÍTICOS}

Todos los bienes materiales provistos por el Estado son bienes económicos, y no libres, por lo que el Mercado por definición tenderá a proveerlos en la medida que sean demandados. Diversos autores se han dedicado a mostrar como bienes tales como las calles $^{13}$ y autopistas ${ }^{14}$ pueden ser provistos por el Mercado. Lo mismo vale para servicios como los de educación, justicia y seguridad. ${ }^{15}$ La razón por la cual no solo el Mercado podría proveerlos sino que sería más eficiente en cuanto a su provisión es la misma por la cual el control estatal total sería inviable ${ }^{16}$ : la ausencia de un sistema de precios que opere como una serie de indicadores de asignación racional de recursos basada en el cálculo económico.

Por otro lado, y como hemos visto, es una contradicción que se definan a los Bienes Públicos como aquellos «No Rivales» $\mathrm{y}$ «No Excluibles». En el primer caso porque el Mercado puede proveer bienes económicos cuyo efecto opere como no rival en el consumo (caso del Faro demostrado por Coase) y por tanto su provisión por parte del Estado sería irrelevante. Y en el segundo porque la mera definición de ciertos bienes como de «No Exclusión» no implica que el Mercado no pueda desarrollar mecanismos

\footnotetext{
13 Ver Block (1983).

14 Ver Block (2009).

15 Ver Rothbard ([1973] 2002).

16 Ver Mises ([1922] 1962).
} 
efectivos de exclusión y a la vez porque este criterio suele referirse a lo que no debería ser excluido, más que a lo que no podría ser excluido. Pero la contradicción se acentúa aún más cuando queda en evidencia que ni siquiera los bienes señalados como Públicos no cumplen con tales características.

Si bien con los bienes materiales es evidente que los mismos son bienes económicos que la teoría económica claramente demuestra que pueden ser provistos con mayor eficiencia por parte del Mercado, algo diferente ocurre con los bienes inmateriales. Como hemos visto anteriormente, los bienes inmateriales pueden convertirse en bienes libres una vez producidos. A su vez, si opera una restricción externa en su consumo, pueden ser bienes económicos nuevamente. En ciertos bienes denominados Bienes Públicos, tales como la Ley, parecería que opera este principio. Una vez que una ley ha sido legislada, opera como una norma o pauta de comportamiento a ser respetada por los ciudadanos de un determinado país. Por lo tanto, no es rival ni excluible en su consumo por parte de uno o más individuos. En tal caso podríamos decir que esta clase de Bienes Públicos es la que más se acercaría a la definición dada. ${ }^{17}$ Sin embargo existen autores que afirman que la producción de leyes sería más efectiva bajo la órbita del Mercado. ${ }^{18}$

Sin embargo observamos que en la generalidad de los casos, aún cuando el Mercado sea más eficiente en la provisión de los bienes señalados como Bienes Públicos, es el Esatdo el que los provee. Este es el caso de la Salud, la Educación, Infraestructura, etc. Por lo tanto, como la definición de Bienes Públicos no les cabe, debemos identificar la razón por la cual el Estado tiende a proveerlos en otras cuestiones que no están relacionadas a los requerimientos objetivos que la definición indica. Y dicha razón es exclusivamente política, no económica. Los Bienes Públicos debieran de ser entonces llamados Bienes Políticos.

\footnotetext{
17 James Buchanan considera a la Ley como un Bien Público, a la vez que un Bien de Capital. Ver Buchanan ([1975] 2009, 161). Podríamos incluso decir, siguiendo la clasificación de los bienes realizada por Carl Menger ([1871] 2007), que la Ley es el Bien de Capital de más alto orden porque al significar el respeto por los derechos de propiedad es aquel bien de Orden Superior que hace que todos los bienes de ordenes inferiores puedan ser utilizados.

18 Ver Friedman ([1973] 1989).
} 
Esto es así puesto que aquellos bienes que el Estado provee no son en su mayoría, como hemos visto, no excluibles y / o rivales en el consumo sino que su provisión se justifica de tal forma. El verdadero criterio de selección de Bienes Públicos es la arbitrariedad de la burocracia, basada en determinadas ideas propuestas por intelectuales sobre el rol del Estado, lo cual los hace Bienes Políticos. Podríamos decir, siguiendo este razonamiento, que si una campaña de propaganda política con una fuerza suficiente se pondría en práctica, podría concebirse que la provisión de calzado llegase a ser considerada por una mayoría como un Bien Público y por tanto eventualmente provista por el Estado.

Así, proponemos la denominación de Bienes Políticos para los denominados Bienes Públicos porque resultarían de un proceso político, y no por razones económicas (supuestamente indicadas por la definición) que obligarían a que sean provistos por el Estado.

\section{$\mathrm{V}$ \\ EL CASO DE LAS EXTERNALIDADES \\ Y LOS FREE RIDERS}

Así observamos que no solo la categoría de Bienes Públicos resulta contradictoria, sino que aquellos bienes que el Estado provee podrían ser provistos por el Mercado con mayor eficiencia. Sin embargo con frecuencia se afirma que en ausencia del Estado en ciertos casos no pueden internalizarse externalidades positivas (con los consecuentes free riders) ${ }^{19}$ o negativas, ${ }^{20}$ por lo que la provisión de los bienes que generen tales externalidades positivas debieran ser provistos por el Estado, mientras que el Estado debería intervenir para internalizar aquellas externalidades negativas que el Mercado no tiene forma de lograr internalizar.

Aún así el Mercado puede encargarse también de solucionar este asunto. La obra de Harold Demsetz, ${ }^{21}$ Ronald Coase y James

19 Como el caso de los faros presentado por Paul Samuelson en su manual de economía Economics, que posteriormente Coase refutaría con evidencia histórica. Ver Foldvary (2003).

20 Ver Coase (1960).

${ }^{21}$ En Demsetz $(1967,1)$. 
Buchanan ${ }^{22}$ (entre otros) consiste en un análisis de cómo los derechos de propiedad se constituyen en una institución que permite responder satisfactoriamente a reducir costos e internalizar externalidades negativas, a la vez que positivas (beneficios). Las externalidades negativas habían sido un objeto de estudio de los economistas desde Pigou (e incluso antes), llegando estos a la conclusión de que el Estado era el agente que debía proceder a internalizar costos a aquellos agentes que los generen (solución a las externalidades negativas).

Sin embargo Ronald Coase demostró, mediante su famoso Teorema, ${ }^{23}$ que no es necesaria la intervención del Estado. El mismo afirma que si los costos de transacción son bajos y los derechos de propiedad están correctamente definidos, entonces es posible solucionar problemas de externalidades mediante el propio acuerdo de los individuos involucrados. Si, por el contrario, los costos de transacción son altos entonces la solución vendrá de la contratación de un árbitro que asigne derechos de propiedad y promueva la internalización de costos a los responsables.

El sistema opera de forma tal que los agente económicos, en la búsqueda de internalizar beneficios, promueven también el arreglo para que los agentes que generan externalidades negativas las internalicen (a los efectos de que los primeros puedan maximizar beneficios). Por tanto existe una auto regulación en el Mercado. Las instituciones que surgen como árbitros de tal proceso son la Firma, el Derecho, e incluso algunos afirmarían que el propio Estado, entre otros. Su rol básico es el de arbitrar la internalización de beneficios y costos.

El análisis de costos y beneficios promueve, por lo tanto, la búsqueda de disminuir costos y a la vez de encontrar formas de reducir externalidades una vez identificadas. La reducción se da a través del perfeccionamiento y expansión de las instituciones antes mencionadas, para hacer uso de las economías de escala una vez que el mercado ha crecido. Si bien la importancia de la asignación de derechos de propiedad ya se encontraba en la famosa teoría de Garrett Hardin, «la Tragedia de los Comunes», este no

\footnotetext{
22 Ver Buchanan, J. ([1964] 1999).

23 Ver Coase (1960).
} 
es más que un desarrollo pequeño del gran estudio realizado por estos autores. ${ }^{24}$

Por tanto podemos observar que el progreso de la sociedad se da en la búsqueda de asignación de derechos de propiedad a los efectos de reducir costos y riesgos. En este sentido, Coase también ha resaltado el rol de la tercerización como mecanismo de eficiencia económica (si bien la Ley de Asociación de Ricardo ${ }^{25}$ lleva implícitamente a la aceptación de este principio antes de que Coase lo formulara).

Es importante notar que este modelo estudiado por los economistas antes mencionados no se basa en uno de los supuestos básicos del modelo de Competencia Perfecta, que es el Conocimiento Perfecto, precisamente porque los agentes deben buscar conocimiento que no tienen a los efectos de asignar derechos de propiedad y solucionar problemas de externalidades. ${ }^{26}$ Cuando se afirma que el Estado debería entrometerse para solucionar dichos problemas implícitamente se supone que posee o podría poseer por defecto un conocimiento que por definición es imposible tener u obtener a menos que los agentes actúen.

El concepto de «Fallos de Mercado», por tanto, deja de tener vigor en la mayoría de los casos, precisamente porque el mecanismo de asignación de derechos de propiedad promueve su solución sin intervención del Estado. En este sentido, las instituciones surgen para reducir los «costos de transacción» que de otra forma harían que los acuerdos entre individuos resultaran o en acuerdos de pequeña escala o inexistentes. Douglas North, ${ }^{27}$ teórico de este estudio, afirma que el rol de las instituciones es precisamente la reducción de dichos costos. Tales instituciones pueden ser, siguiendo a Hayek, Cosmos (orden espontáneo, es decir que

\footnotetext{
24 Ver Ostrom ([1990] 2002, 2).

25 Ver Mises ([1949] 1986, 251-258).

26 En este contexto, un ejemplo del Análisis Económico del Derecho es ilustrativo: A la hora de efectuar sus fallos, los jueces deben tender a promover el funcionamiento correcto del sistema en una escala global. En este sentido, el Juez no puede saber lo que es justo, sino lo que no lo es (desde una perspectiva Popperiana aplicada al Derecho). Podríamos decir, en este sentido, que el refinamiento del concepto de justicia se da en las sucesivas repeticiones del juego.

27 Ver North ([1986] 1990).
} 
surgen a través del proceso de interacción social) o Taxis (orden creado, indicando un orden diseñado deliberadamente). Sin embargo es importante recalcar que no es lo mismo un Taxis que se cree para promover el orden (tal como el Estado), que una regulación arbitraria. Un ejemplo de Cosmos es la Empresa (o la Firma).

La obra de Mancur Olson ${ }^{28}$ y Elinor Ostrom es también relevante a este respecto puesto que ambos estudiaron la forma en que el propio interés (basado en los derechos de propiedad) de los miembros de un grupo, genera que estos solucionen problemas de «Bienes Públicos» y la manutención de recursos comunes. Especialmente la obra de Ostrom muestra que aún en comunidades primitivas (como el caso indígena), mecanismos de preservación de derechos de propiedad arribados espontáneamente permiten la solución a problemas como los antes planteados.

En su obra, Ostrom logra identificar que bajo la definición de límites de exclusión sobre los recursos, sumado a la determinación de reglas por parte de la comunidad, se torna posible la preservación de los mismos bajo propiedad comunal. Así también es relevante que los propietarios determinen normas de uso y disposición de los recursos, siendo el control sobre los mismos la garantía de respeto de dichas normas (estableciendo a su vez sanciones a los infractores). Entre otras cuestiones, tales como la organización de control de los recursos bajo distintos niveles (con la propia comunidad como el más básico), podemos observar que lo que Ostrom describe son arreglos institucionales que surgen de forma espontánea y se perfeccionan en un proceso de prueba y error. Así, la propiedad comunal es una forma a través de la cual es posible la obtención de bienes que se definen rivales pero de difícil exclusión, y a partir de esta se podrían analizar alternativas de control sustentables sobre los recursos (no solo en términos económicos y políticos sino también ecológicos).

La interrelación, por tanto, entre Derecho y Economía, aparece directamente puesto que autores como Demsetz consideran que el comercio no es otra cosa que el intercambio de paquetes de derechos de propiedad.$^{29}$ En este sentido es importante notar que no

\footnotetext{
28 Ver Olson ([1965] 1971)

29 Ver Demsetz $(1967,1)$.
} 
es el comercio el que hace al derecho (si bien se retroalimentan), sino el derecho quien hace al comercio. Precisamente porque se daría un reconocimiento vertical de los derechos, siendo el Constitucional primero, posteriormente el derecho de propiedad, y finalmente así se daría el reconocimiento de la legitimidad de las transacciones realizadas y sus efectos en los patrimonios de los involucrados. Dicho estudio sería elaborado por James Buchanan, quien se enfocaría con mayor atención en el análisis económico de la Política y las decisiones políticas por parte de la ciudadanía.

El respeto por los derechos de propiedad surgiría a través de un «juego repetido» (Implicancia de North), donde una de sus causas es la llamada «Noción de Justicia» y el sentimiento de Justicia. Si bien Buchanan no le prestaría atención a la Justicia y si al Poder, este no deja de ser un concepto interesante y relevante a la hora de estudiar este proceso. Esto es así ya que si bien tanto Buchanan como Demsetz se concentran en identificar externalidades y estudiar casos como los conocidos de la alambrada (y el ganado), los llamados Bienes Públicos, los free riders y demás; el concepto de Justicia es esencial para los agentes que comercian.

Como ha afirmado Coase, y siguiendo lo antedicho sobre el Estado como un Taxis que promueve el orden, la regulación política puede solucionar los problemas de costos de transacción en ciertas circunstancias, pero debe tenerse en cuenta (advertencia típicamente Hayekiana) que con ello se crea una nueva estructura de incentivos que puede terminar por alterar la situación y generar peores consecuencias que el problema inicial que se pretendía solucionar.

Buchanan, como hemos dicho, se concentró con mayor atención en el estudio del poder y las estructuras de poder, fundamentalmente bajo la democracia. En el análisis económico de la política, Buchanan demuestra que las «Fallos del Estado» terminan siendo peores que las «Fallos del Mercado». Tal conclusión está implícita en la obra de Hayek, donde la única forma de coordinación del conocimiento disperso (que es el Mercado), se ve intervenida por un ente, el Estado, que lo único que hace (por qué es lo único que puede hacer a este respecto) es bloquear el proceso de transmisión de información y alterar el delicado esquema de incentivos que se construye espontáneamente en la sociedad a través del proceso de coordinación del mercado (basado en el sistema de precios). 


\section{VI \\ LA DEMOCRACIA NO REPUBLICANA \\ COMO MARCO DE EXPANSIÓN \\ DE LOS BIENES POLÍTICOS}

Siguiendo la obra de Buchanan (la Escuela de la Elección Pública), resulta claro identificar que en un marco democrático sin una República que garantice el respeto por los derechos individuales bajo una constitución fuerte, diversos grupos de interés intentarán atraer privilegios por parte del Estado. En este contexto la expansión de los Bienes Políticos (justificados mediante la categoría de Bienes Públicos) resulta clara, precisamente porque el sistema opera de forma tal que no solo permite, sino que promueve la expansión estatal.

El estudio de Buchanan ${ }^{30}$ lleva al reconocimiento de las realidades y vicisitudes del sistema democrático. El mismo llega a la conclusión de que a la ciudadanía le resulta irrelevante el voto, puesto que es uno sobre millones, y el efecto del voto personal resulta marginal. Por tanto el costo de oportunidad de dedicar tiempo al estudio de la política resulta alto (el esquema de costo/beneficio es claramente negativo). A su vez resulta difícil comprender las ideas de los políticos a la luz de las falsedades que se difunden por doquier, mientras el discurso político y la propaganda pretenden describir propuestas positivas. Así, la racionalidad del votante (en el sentido de tomar una decisión sin demasiada meditación debido al alto costo de oportunidad) es bastante limita$\mathrm{da}$, teniendo en cuenta que lo anterior le lleva casi con seguridad a no tomar la decisión correcta.

A esto debemos sumarle otros factores que disminuyen aun más el compromiso ciudadano, tales como la voluntariedad del voto (en el caso de Estados Unidos, donde Buchanan estudia) ${ }^{31}$ y el voto realizado en «conjuntos» de candidatos, donde ni siquiera se conoce quienes son la mayoría. A su vez se suele caer en simplismos donde se creen en las propuestas de los políticos como si

\footnotetext{
30 Ver Buchanan, J. ([1975] 2009).

31 Esto no es aplicable al caso Argentino en particular o Latinoamericano en general.
} 
consistirían en magia, debido precisamente a que son identificables, en contrapartida a la acción invisible e impersonal del mercado. Si los políticos proponen soluciones se identifica directamente que hay un plan y una planificación, independientemente de los resultados posteriores (que cuan mayor complejidad tenga el problema a solucionar, mayor probabilidad de que la intervención falle), mientras el mercado resulta impersonal e invisible, por lo tanto poco eficiente a la luz de los votantes (aun cuando fuese la verdadera solución, o la más cercana). El costo de aprender el funcionamiento de una economía de mercado no parecería ser inferior al beneficio por el hecho de votar correctamente, pero aún así en el cálculo individual no se suele tener en cuenta.

La visión de los políticos como una familia y al Estado como el hogar es otra fuente de irracionalidad en la democracia, siendo la constitución del famosos Estado Paternalista. Igual situación se identifica con respecto al léxico político, donde se denominan a ciertos recursos como «Nacionales» y siguiendo la lógica anterior (donde el Estado «somos todos»), de propiedad de todos los ciudadanos. Por lo tanto estos se constituyen discursivamente en Bienes Públicos, donde el estado mantiene su control y quienes lo componen (o a quienes estos los concesionan) se hacen de los beneficios de la renta monopólica que les es característica. El discurso político es fundamental a la hora de moldear las decisiones de los votantes en base a la manipulación del lenguaje.

La diferencia en el interés de los resultados de la votación es también relevante, puesto que quienes tienen intereses en la continuidad de cierta política (como los beneficiarios de programas de asistencia social, o aquellos que pertenecen al llamado «Voto Cautivo») no votarán de la misma forma que un joven que no conoce nada de política o un anciano analfabeto. La ausencia de sistema de precios imposibilita la asignación de los puestos políticos en relación a las preferencias de los consumidores (en el sentido de que no se sabe si los elegidos promoverán el funcionamiento correcto de la economía en relación a las preferencias individuales) y deja a los ciudadanos a merced de un lenguaje político engañoso y de una propaganda inescrupulosa.

Siguiendo el ejemplo anterior, el mercado (definido como una verdadera democracia), opera de forma tal que la gente obtiene 
lo que desea en relación a su mérito en el servicio a los demás (debido a que compra en base al ingreso que obtiene de su trabajo), mientras en política quedan subyugados por la decisión mayoritaria. En el mismo sentido, mientras en el mercado los cambios son tan rápidos como rápidamente cambien las decisiones individuales de los agentes, en política los tiempos están fijos y determinados arbitrariamente. En tal sentido, el único mecanismo para que los cambios se den con mayor rapidez es el de los llamados «lobbystas» que presionan a las autoridades políticas a los efectos de lograr cambios en su beneficio, convirtiendo a la sociedad en una lucha de grupos de interés donde los más grandes imponen su peso a los más pequeños bajo el paraguas defensor del Estado, que opera como un agente sujeto al cohecho y la manipulación. Con la consiguiente violación de los derechos individuales por la erosión de las instituciones y el despilfarro de recursos de los contribuyentes, la sociedad (y más aún, la civilización) se deteriora.

Precisamente son las minorías organizadas las que terminan explotando a las mayorías desorganizadas si toman control del estado a través de arreglos con el poder político de turno, a través de barreras arancelarias que perjudican al consumidor local, impuestos, exenciones fiscales, zonas monopólicas concesionadas, subsidios, etc. En este sentido los impuestos dejarían de ser el pago por la contraprestación del servicio de seguridad y justicia (la llamada función básica del Estado), y pasan a ser una arbitrariedad.

En este contexto, la única forma de limitar el poder es mediante una Democracia con República, amparada por el respeto de una constitución como Ley fundamental. ${ }^{32}$ De lo contrario las minorías se ven superadas por las mayorías y la expoliación, bajo la expansión de los Bienes Politicos justificados en el discurso político como Bienes Públicos, alcanza proporciones cada vez mayores. Si bien varias propuestas son desarrolladas por Hayek (quien se centra en el sistema de gobierno) ${ }^{33}$ y Buchanan ${ }^{34}$ (quien prefiere concentrarse en la figura de la

${ }^{32} \mathrm{El}$ concepto al que hacemos referencia es el de Rule of Law, o Imperio de la Ley.

33 Ver Hayek ([1982] 1998).

${ }^{34}$ Con respecto a la Constitución y los límites que esta puede establecer, a modo de ejemplo veamos la afirmación de Thomas Jefferson en una carta a John Taylor con 
Constitución como garantía del respeto por los derechos individuales), si no se reconoce la contradicción inherente al concepto de Bienes Públicos no es posible reconocer la causa de la expansión estatal, con el consecuente deterioro institucional.

\section{VII ¿EXISTE UN MARCO DE APLICACIÓN PARA EL CONCEPTO DE BIENES PÚBLICOS?}

Como hemos visto el concepto de Bienes Públicos es contradictorio y resulta más útil referirse a Bienes Políticos, a la vez que en base al estudio de los autores anteriormente mencionados, queda claro que el Mercado podría proveer con mayor eficiencia aquellos bienes que hoy provee el Estado. Sin embargo podemos identificar que algunos bienes, aun cuando no responderían a la definición de Bien Público (puesto que, como hemos visto, haría contradictorio que sean provistos siquiera por el Estado), si podrían ser inherentes a las funciones del Estado ${ }^{35}$ siguiendo la lógica de la economía expresada en los estudios anteriores.

Para ello debemos analizar los conceptos Hayekianos de Cosmos y Taxis. En el análisis Hayekiano se distinguen dos tipos de órdenes ${ }^{36}$ posibles para la configuración de los fenómenos: espontáneos y creados o artificiales. ${ }^{37}$ Los órdenes creados son configurados por uno o varios diseños exógenos al fenómeno, y por tanto son simples, esto es, al ser creados por la mente humana pueden ser comprendidos por ella (y regulados y controlados por la misma). Ellos pueden ser estudiados en su totalidad y a la vez

\footnotetext{
respecto a la Deuda Pública: «I wish it were possible to obtain a single amendment to our Constitution. I would be willing to depend on that alone for the reduction of the administration of our government to the genuine principles of its Constitution; I mean an additional article, taking from the federal government the power of borrowing» (26 de Noviembre de 1798). En Straub $(2012,26)$.

35 Algunos podrían llamarlos Bienes Públicos, pero en sentido amplio (implicando bienes económicos pero con efectos típicos de bienes libres). Esto es así puesto que en sentido estricto, la definición incurre en una contradicción.

36 Siendo orden un conjunto de partes integradas.

37 Ver Hayek ([1982] 1998, 35).
} 
resultan de fácil identificación, precisamente porque han sido creados. Los mismos son, definidos por Hayek, como un Taxis.

En sentido contrario los órdenes espontáneos surgen no de la voluntad de específicos individuos, sino que resultan de un proceso en el cual sus partes intervinientes lo crean inadvertidamente (de creación endógena). Por tanto no pueden identificarse fácilmente, y su estudio requiere el análisis de partes del mismo a los efectos de intentar comprender el todo. Por otro lado el mismo se auto regula a través de las pautas de comportamiento de las cuales evolucionó y que se constituyeron en regularidades. Precisamente porque no puede comprenderse cabalmente el origen y funcionamiento de tales ordenes, intentar convertirlo en una organización $^{38}$ resultará fútil, justamente porque el mismo funciona con sus propias reglas. Aún cuando pueda controlarse una parte, al no poder realizarse un estudio integral de tales fenómenos, surgirán consecuencias no planeadas que alteraran indefectiblemente los planes originales. Hayek define a estos fenómenos como un Cosmos.

La sociedad, como un fenómeno de orden espontáneo, se coordina fundamentalmente a través del conocimiento que sus miembros poseen y transmiten entre si, al cooperar a través de diversas instituciones que surgen también espontáneamente. Las mismas, tales como la moral, el derecho, el lenguaje y demás, permiten incrementar la cooperación, el intercambio y por tanto el progreso, a la vez que tornan el fenómeno social en uno aún más complejo.

Por lo tanto cada vez que se intenta intervenir en un orden espontáneo se generaran «consecuencias no intencionadas» que implicarán desajustes y descoordinación. Esto es así puesto que se está operando sobre un fenómeno complejo del que no se pueden extraer las variables relevantes intervinientes a los efectos de atenuar las consecuencias negativas de las intervenciones. De ahí que la intervención estatal en la economía genere desajustes, mal asignaciones de recursos, y finalmente pobreza. La sociedad no es un laboratorio del que puedan aislarse elementos como en las ciencias naturales.

38 Otro sinónimo de orden creado. 
El conocimiento sobre el estudio de ambos fenómenos es, por lo tanto, diferente..$^{39} \mathrm{El}$ conocimiento de órdenes espontáneos está disperso, por lo que nadie puede centralizarlo ni saber con exactitud su origen. A su vez suele ser subjetivo y práctico, por lo que no necesariamente podrá ser articulado en proposiciones bajo un sistema que siga razonamientos unidireccionales (por ejemplo cuales serán las consecuencias de establecer un sistema de Common Law en la Argentina). Los órdenes espontáneos se basan en comportamientos que dan lugar a hábitos en procesos evolutivos que no pueden ser catalogados o formalizados, sino que se convierten en conocimiento tácito. ${ }^{40}$

A diferencia del orden espontáneo, el orden creado si es pasible de toda esta articulación. El conocimiento científico es, por ejemplo, un orden creado. Esto es así puesto que el mismo esta formalizado, tiende a la centralización y a su vez se encuentra articulado. ${ }^{41}$

En este sentido, Hayek hace una distinción en cuanto al estudio de las Ciencias. Divide a las mismas en Naturales y Sociales, siendo que las primeras estudian fenómenos simples, cuyo objeto de estudio son entes fuera de la mente del sujeto, de observación directa, que identifican regularidades y constantes y cuya modelización se basa en relaciones funcionales. En sentido inverso, las segundas estudian fenómenos complejos, donde su objeto de estudio son ideas que requieren ser interpretadas mediante teorías previas, a la vez que no se corroboran constantes sino que predominan las variables y las relaciones entre los fenómenos no son como aquellas en Ciencias Naturales. En este sentido es evidente la influencia del pensamiento de su maestro, Ludwig von Mises, por ejemplo en la distinción realizada por este último de Probabilidad de Clase y Probabilidad de Caso, ${ }^{42}$ siendo que en Ciencias Sociales pueden encontrarse regularidades solo

\footnotetext{
39 Ver Huerta de Soto (2012, 15-50).

40 Ver Huerta de Soto ([1992] 2001).

41 Algunos autores disienten con esta visión, puesto que afirman que el conocimiento científico depende de cuestiones sociales, ideológicas, y demás que no necesariamente se limitan estrictamente al método científico y corroboraciones con un status epistemológico riguroso.

42 Ver Mises ([1996] 1949).
} 
en las clases de entes estudiados, pero no predecir casos particulares (de ahí la posibilidad del funcionamiento de la industria de seguros).

Hayek ataca ${ }^{43}$ a quienes identifica como aquellos que sostienen la Razón como una herramienta que puede alcanzar la omnisciencia (de raíz Cartesiana), o los por el llamados Cientistas. La Razón es una herramienta de cognición, pero no puede conocer todo. En este sentido lo que Hayek intenta es apelar a argumentos racionales para ilustrar la imposibilidad del conocimiento absoluto. Cualquier intento de controlar todo desde el poder del Estado lleva al totalitarismo. ${ }^{4}$

La clasificación de órdenes en Cosmos y Taxis obliga al científico a tener la humildad suficiente como para intentar estudiar con cautela los mismos. Y si bien un Taxis puede convertirse en un Cosmos y viceversa, esto no quiere decir que el Cosmos pueda planificarse centralmente, sino que este será resultado de un proceso evolutivo que tendrá una cantidad de variables intervinientes lo suficientemente complejas como para no permitir distinguir el origen y funcionamiento total del mismo. ${ }^{45}$

Por lo tanto surge la pregunta: ¿El Estado es un Cosmos o un Taxis? La respuesta tendrá que ver con la explicación histórica que se elija. Sin embargo la teoría económica puede decirnos mucho al respecto. Si el Estado es una agencia que tiene el monopolio del uso de la fuerza física represiva, y que solo puede usarla (en consonancia con una Constitución) contra aquellos que inician su uso, entonces su función básica es la de proveer Seguridad y Justicia. Justicia para proteger los derechos individuales de los ciudadanos en las disputas que surgieren entre ellos, y seguridad para proteger sus derechos individuales frente al

43 En Hayek ([1982] 1998, 8).

44 Ver Hayek ([2010] 1944).

45 Quienes sostienen la validez de esta visión de la sociedad pero al mismo tiempo reconocen la necesidad de efectuar cambios bajo la guía de planes centrales, los mismas deberían (si desean tener en cuenta las limitaciones que dificultan actuar sobre órdenes espontáneos) seguir un lineamiento como el de Karl Popper de «Ingeniería Social Gradual» ([1945] 2010, 173-184). No intervenciones generales e integrales cuyas consecuencias no intencionadas podrían ser potencialmente de una magnitud mayor («Ingeniería Utópica»). 
inicio del uso de la fuerza de unos contra otros o de extranjeros contra ellos. Por tanto el Estado es una agencia de seguridad monopólica.

Si el Estado es un Cosmos entonces considerarlo un Taxis y promover su desaparición generaría «consecuencias no intencionadas»; más si es un Taxis entonces podría concebirse su reemplazo por agencias de seguridad privadas en competencia. Aquí es relevante la visión de Robert Nozick al respecto, puesto que el afirma que en un estado de anarquía con agencias de seguridad en competencia, estas tenderían a desaparecer a favor de una que finalmente monopolice la provisión de seguridad y justicia. Por lo tanto esta se convertiría en el Estado. Así, el Estado sería un Cosmos (si bien su constitución final y organización serían un Taxis) que surge a raíz de una necesidad objetiva de la economía: las economías de escala. Es más eficiente que una sola agencia provea ese servicio, y sería perjudicial al propio Mercado que existiesen varias disputándose esa función (puesto que esto implicaría un derroche de recursos).

En este sentido podríamos afirmar que las funciones básicas del Estado bajo el esquema liberal clásico pueden considerarse funciones legitimas a la luz de la teoría económica y las necesidades objetivas de una economía real. En tal sentido, la Ley operaría entonces como un bien cuya creación por parte de legisladores, una vez crea$\mathrm{da}$, funciona como un bien cuyas características son aquellas de los bienes libres (en el sentido de Mises descripto más arriba; a su vez lo expresado por Buchanan). Por lo tanto si existe solo una agencia de provisión de seguridad y justicia (el Estado) no hay rivalidad y exclusión en el consumo de tal bien. De hecho la evolución de la Ley (en el sentido del Derecho bajo un esquema de Common Law como un orden espontáneo) requiere que esta misma sea utilizada por uno o más individuos (por ejemplo jueces para aplicarlo en la definición de derechos de propiedad y resolución de litigios) para que la misma se refine y así la institución del Derecho progrese.

Aún teniendo en cuenta esto, la categoría de Bienes Públicos no es correcta para definir las funciones del Estado, sino que estas deben ser determinadas en base a aquellas actividades que, no es que el Mercado no provea, sino que por economías de escala 
en un proceso evolutivo, este tercerizó en el Estado como el árbitro que soluciona los problemas relativos a servicios de seguridad y justicia. Aquellos autores que afirman que ciertas instituciones, como la Ley (entendida como Nomos) ${ }^{46}$ deberían ser catalogadas como Bien Público erran puesto que, aplicando la Navaja de Ockham, ${ }^{47}$ no es necesario crear una categoría adicional a la de institución. Esto solo legitimaría la categoría de Bienes Públicos y al mismo tiempo resultaría irrelevante, puesto que entender la Ley como una sub-categoría de la categoría de Bienes Públicos, cuando al mismo tiempo no existe otra sub-categoría ni una definición específica para la categoría principal, entonces se estaría creando un ente innecesario. Podríamos decir que, siguiendo a Buchanan, la Ley si es un Bien de Capital (al igual que otras instituciones fundamentales de la civilización, como el lenguaje y la moral), más la categoría de Bien Público no es ni relevante ni apropiada para definirlo.

La categoría de Bien Público no solo es contradictoria, sino que en realidad consiste en Bienes Políticos, en el sentido de que su provisión por parte del Estado no surge de ninguna necesidad objetiva de la economía (puesto que el Mercado supuestamente no proveería tales servicios), sino por un proceso de presión política y arbitrariedad burocrática en la delimitación del accionar estatal. Por ello, como hemos visto en el presente trabajo y tal como afirma Nozick, ${ }^{48}$ el máximo Estado justo (y económicamente eficiente) es el Estado Mínimo.

\section{REFERENCIAS BIBLIOGRÁFICAS}

Buchanan, J. ([1964] 1999): «The Demand and Supply of Public Goods» en The Collected Works of James M. Buchanan. Volume 5. Indianapolis: Liberty Fund.

- ([1975] 2009): Los Limites de la Libertad - Entre la Anarquía y el Leviatán. Madrid: Katz; Liberty Fund.

\footnotetext{
46 Ver Hayek ([1982] 1998).

47 Afirma que «las hipótesis no deben multiplicarse sin necesidad».

48 Ver Nozick ([1974] 1999, ix).
} 
Block, W. (1983): «Public Goods and Externalities: The Case of Roads» en The Journal of Libertarian Studies. Vol. VII. N.o 1 (Primavera): pp. 1-34. Disponible en http://mises.org/ journals/jls/7_1/7_1_1.pdf.

- (2009): «The Privatization of Roads and Highways - Human and Economic Factors». Auburn: The Ludwig von Mises Institute.

Conse, R. (1960): «The Problem of Social Cost» en The Journal of Law and Economics. Vol. III (Octubre): pp. 1-44.

Demsetz, H. (1967): «Hacia una Teoría de los Derechos de Propiedad». Traducción de American Economic Review (Mayo). Disponible en http:/ / www.eumed.net/cursecon/textos / Demsetz_teoria-derechos-propiedad.pdf .

Foldvary, F. (2003): «El Faro como un Bien Público del Sector Privado»; Trabajo de Investigación N.․ 46. El Instituto Independiente. Disponible en http://www.elindependent.org/ articulos/article.asp?id=757 .

Friedman, D. ([1973] 1989): The Machinery of Freedom - Guide to Radical Capitalism. Disponible en http:/ / www.daviddfriedman. com/The_Machinery_of_Freedom_.pdf.

HAYEK, F.A. ([1944] 2010): Camino de Servidumbre. Madrid: Alianza Editorial, S.A.

- ([1982] 1998): Law, Legislation and Liberty, Vol. 1 Rules and Order, Vol. 2 The Mirage of Social Justice, Vol. 3 The Political Order of a Free People. Great Britain: Routledge.

- ([1988] 1992): The Fatal Conceit. The Errors of Socialism. Great Britain: Routledge.

Hoppe, H.H. (1989): «Fallacies of the Public Goods Theory and the Production of Security» en The Journal of Libertarian Studies. Vol. IX. N. 1 (Invierno): pp. 27-46. Disponible en http://mises.org/journals/jls/9_1/9_1_2.pdf.

Huerta De Soto, J. ([1992] 2001): Socialismo, cálculo económico y función empresarial. Madrid: Unión Editorial, S.A.

- (2012): La Escuela Austriaca - mercado y creatividad empresarial. Madrid: Editorial Sintesis.

DE JASAY, A. ([1985] 1998): The State. Indianapolis: Liberty Fund. Menger, C. ([1871] 2007): Principles of Economics. Auburn: The Ludwig von Mises Institute. 
Mises, L. VON ([1922] 1962): Socialism: An Economic and Sociological Analysis. New Haven: Yale University Press.

- ([1949] 1986): La acción humana - Tratado de economía. Madrid: Unión Editorial.

- ([1949] 1996): Human Action. San Francisco: Fox \& Winkles. Murphy, R. ([2002] 2010): Chaos Theory - Two Essays on Market Anarchy. Auburn: Ludwig Von Mises Institute.

NorTH, D. ([1986] 1990): «La Nueva Economía Institucional» en Revista Libertas 12, Instituto Universitario ESEADE (Mayo). Traducción del Journal of Institutional and Theoretical Economics, Vol. 142. Disponible en http://www.eseade. edu.ar/files/Libertas/33_5_North.pdf .

NozIck, R. ([1974] 1999): Anarchy, State and Utopia. Cornwall: Blackwell.

Olson, M. ([1965] 1971): The Logic of Collective Action - Public Goods and the Theory of Groups. Cambridge: Harvard University Press.

Ostrom, E. ([1990] 2002): Governing the Commons - The Evolution of Institutions for Collective Action. New York: Cambridge Univesity Press.

Popper, K. ([1945] 2010): La sociedad abierta y sus enemigos. Barcelona: Ediciones Paidós.

RothbaRD, M.N. ([1973] 2002): For a New Liberty. New York: Coller Macmillan Publishers.

SAmuelson, P. ([1969] 1991): "Pure Theory of Public Expenditure and Taxation», en The Collected Scientific Papers of Paul A. Samuelson. (Originalmente en J. Margolis and H. Guitton, eds. «Public Economics: An Analysis of Public Production and Consumption and their Relations to the Private Sectors: Proceedings of a Conference held by the International Economic Association». London: Macmillan.) Massachusetts Institute of Technology: M.I.T. Press: pp. 492-517.

Straub, S. (2012): «The Essential Thomas Jefferson». The Federalist Papers Project. Disponible en http:/ / www.thefederalist papers.org/wp-content/uploads/2012/08/The-EssentialThomas-Jefferson.pdf. 